\title{
Segmentation and Classification of Bi-Rads Medical Images with the Imaging Biomarkers According To Level of Detail
}

\author{
Ratko IVKOVIĆ, Mile PETROVIĆ, Branislav DAKOVIĆ, Branimir JAKŠIĆ, Ivana MILOŠEVIĆ
}

\begin{abstract}
Segmentation is one of the latest directions of digital imaging development, presented by partial segments which are parts of the same image. The currently used algorithms are rare and far from ideal. Depending on the situation, algorithms behave differently, so it is almost impossible to single out a unique conclusion. This paper gives a proposal for new segmentation techniques with the help of Imaging Biomarkers for the digital image processing with auxiliary Hybrid edge detection algorithm and especially a defined threshold of detection. Matlab software is used for giving a particular conceptual design of a partial display of images and for correlating adequate numerical methods of digital processing. The study results confirm the quality of the proposed algorithm and position it for practical use in classification of BI-RADS breasts classified by density.
\end{abstract}

Keywords: BI-RADS medical images; digital Image; imaging biomarkers; level of detail; segmentation

\section{INTRODUCTION}

Precise detection of object's edges is one of the most important parts of digital image processing [1]. Segmentation represents the task of digital image processing in definition of coherent regions inside a digital image [2]. That definition has to come out of one of the already defined parameters. Regarding the field of application of digital image processing, different parameters will be defined [3]. Medicine is a field in which biological parameters will be defined, regarding to which image segments will be isolated. It has to be noted that the parameters were defined based on a quantitative analysis previously done by medical doctors [4]. One of the main methods of edge detection is based on model of active contours, or snake segmentation [5]. Method of active edge detects specific elements of the image, and dynamicity fixates edges of the structure, while minimizing segments entropy. There are several issues related to some implementations of this method. First, it is hard to define the starting pixel in snake initialization. Secondly, the method is dependent on image quality, so images with lower resolution, weak contrast or increased noise have problematic edge detection. Third problem can be found in synchronization method in certain parts of the image and overlapping structures. The listed issues lead to edge detection difficulties. For example, the angle from which the image is generated can be a great problem because different positions of image creation can lead to image edges detection error, and that can lead to image segmentation error. Biological contrast can also produce an error in useful information detection. Finally, the method by itself is complicated because a few parameters need to be defined before iteration. Ergo, it would be useful to develop a nonparametric active contour method, but to this day, only a few of such approaches have been taken [6-8] In manuscript [6], nonparametric model turns the problem of parameter search into a problem of density estimation. Disadvantages of this approach include the selection of core size, and the risk of edge concavity not being found. The problem of density estimation can be connected to the image of biomarkers (BI) and something that already exists as a biomarker can be used; that is the category of the thorax density. Besides this theoretical framework, which is needed to understand the new algorithm, this work has given a methodological framework for its realisation as well as the type of work in different real situations. A proposal for new digital image segmentation techniques by using Hybrid edge detection algorithm, RGB colour model, alpha composite process, full grayscale and a combination of parallel and sequential edge detections is given in this article. One of the main innovations in the proposed image segmentation technique is the central role of the edge detection algorithm. The complete proposed solution is fully compatible with other edge detection algorithms, so it is adjustable for particular situations. So, for example, if there is a new algorithm which gives better results, it is possible, without great modifications, to replace it with that new algorithm, and by doing that, not to affect the quality of other steps in the segmentation done by the suggested method. Each of the algorithms contained in the final algorithm for segmentation has been suggested on the basis of its positive results.

Ergo, positive effects of the laid out solution overcome the laid out defects and difficulties which are created while applying old models of segmentation. New model of medical image segmentation has been laid out with the help of hybrid edge detection and imaging biomarkers with nonparametric method. Its advantages are maintained in elimination of errors formed during noise occurrence in medical images, which is kept as the main characteristic of Hybrid model. In the same way, the model of edge detection gives user an ability to define the detection threshold and takes original edge detection into account, negative and the original image, after application of supplementary algorithm. This kind of algorithm detection, used as a basis, gives a precision in defining of useful information by $93 \%$, as studies have shown. In the same way, area Levels of Detail (LoD), based on thorax density, were defined. It has been shown that already existing classes of thorax density completely correspond with the measurement Level of Detail in the new segmentation method. The speed of image generating is identical to other methods so the attached method can be seen as the original. 


\section{THEORETICAL METHOD \\ 2.1 Hybrid Edge Detection}

In the process of image creation, optical sensor and converting the visible spectrum into electrical signal should be taken into account. That, for now, still imperfect process of image creation of digital image creates certain types of noise, which are differently defined, based on applied optical sensor type. CCD and CMOS sensor differences are well known, as well as algorithm for edge detection in the image creation, as it has been described in the paper [9]. During application of any algorithm in the process of detection, noise matters as well, so algorithms with low threshold of detection represent the noise as an edge. All the given reasons were the basics for filter usage which will "prepare" the image for detection. In other words, this filter has the duty to eliminate $5 \%$ of the noise in digital image, while having a high degree of similarity to the original image, according to the method of SSIM (The Structural SIMilarity) [10-13]. Based on the paper [14], which collects different methods of noise elimination, it can be concluded that Weighted algorithm method suits the assumed initial conditions of image preparation for edge detection. The next step should be the definition of threshold of detection that the user will give. That way the user will have the entire $8 / \mathrm{bit}$ scale. Such a procedure gives a broad detection scope, i.e. defines the sensitivity of detection, so it gives tremendous possibilities. Threshold of detection is defined as difference in values between the two neighbouring pixels. Something like that, in the case of Hybrid model of edge detection, is done through threeway comparison (Fig. 1) of neighbouring pixel values.

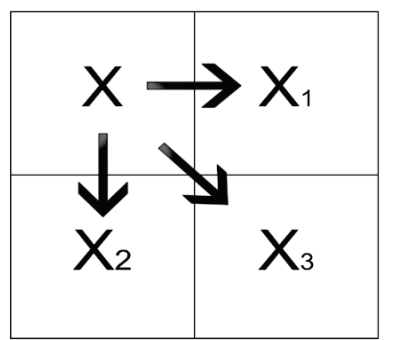

Figure 1 System of pixel values comparison regarding the defined detection threshold

It is important to do a comparison of this type for the negative as well and totalize with the obtained values from the first algorithm. By this procedure, mistakes are conditioned by edge detection, and in the same way, detection is done in the opposite direction, i.e. the detection is now done in direction of $180^{\circ}$ based on starting direction. Third condition is the influence of different illumination levels. For this goal, histogram equalization will be used as supplementary algorithm.

After application of supplementary algorithm, an image whose level of brightness will be almost identical to 8-bit system, as has been shown in Fig. 2, will be obtained. Digital image in this form makes an even bigger difference in values of neighbouring pixel and because of that it is useful for further editing. Application of cumulative histogram in this case gives the most useful results, which is affirmed by many works based on extraction of useful information from the image, to which almost the complete spectre in one mutual part of the scale is illuminated [15].

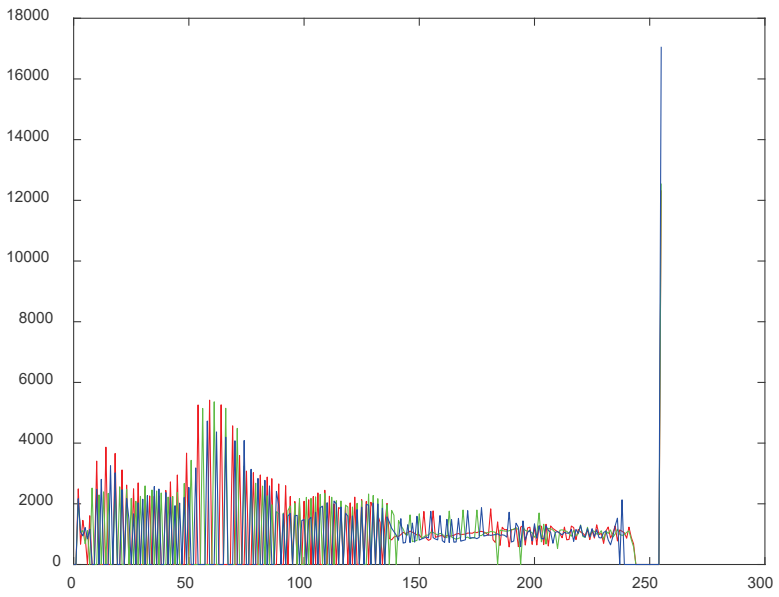

Figure 2 Image histogram after implementation of the supplementary algorithm

Complete algorithm of edge detection by using the Hybrid method is shown in Fig. 3.

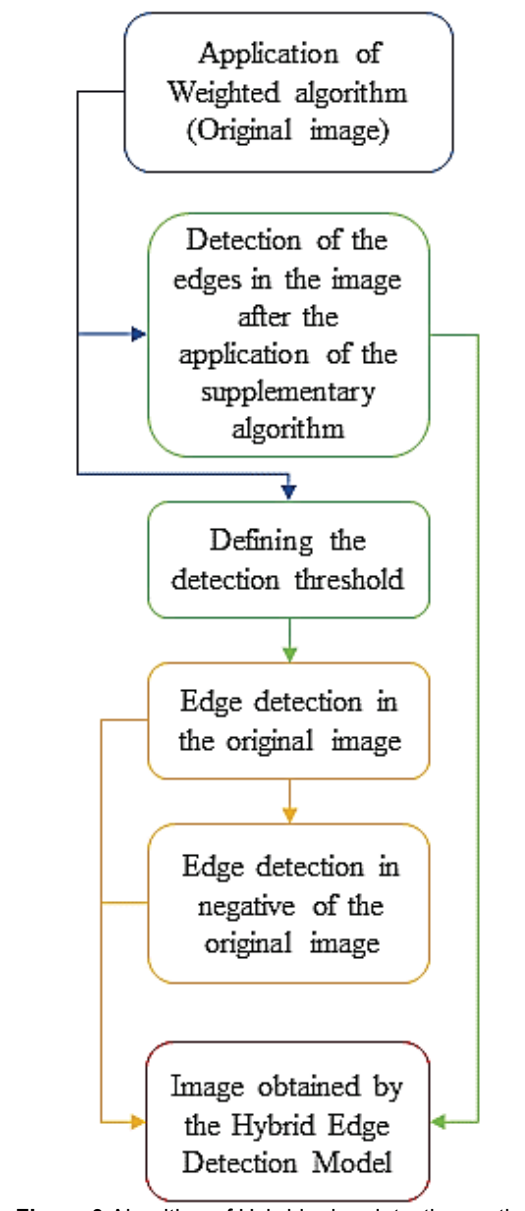

Figure 3 Algorithm of Hybrid edge detection method

After application of Weighted algorithm, in which the noise is eliminated, the image is used as detection basis (original image). Next step is detection threshold definition which is done by user and represents the basics for detection. Detection is done in three completely different steps:

1) On the original image,

2) On the negative of the original image,

3) On the original image after supplementary algorithm application. 
All three received signals are kept as inter-results, and the final result is the image represented by the cumulative sum of the results after three defined detections. By application of the algorithm from the field of digital image which has filtrating as its basics, and then the algorithm which manipulates the level of brightness in the image, and taking into consideration that the final result is shown as a sum of three signals, the complete process can be named the Hybrid method for edge detection. It is necessary to point out that each algorithmic block is independent from the others and in order to get better results, it is possible to change them.

\subsection{Segmentation}

Segments can be defined as a region between certain defined discontinuities in the image. Discontinuities when it comes to digital editing of the image must be shown in a certain mathematical method, which we call edge detection. When it comes to medical images, image segments must represent and describe a certain condition and it is desirable in idealistic cases that after that, the medical treatment of the segment is defined as well. Two standard methods of edge detection, parallel and sequential, do not give relevant results. Parallel way of detection is not ideal because it defines independence of a set of points that are located on the edge, whether the point stays at the edge or not. Sequential detection of the edge is based on a point and the entire result of this process is based on the well-chosen point. That is why the Hybrid method is the best option for segmentation of this type because it compares and takes into realization the interresults from a few series of elementary image processing.

\section{IMAGING BIOMARKERS (BI)}

Definition of characteristics of an image, which points out biological processes or responds to interventions, is called Imaging Biomarkers BI. Based on defined states of $\mathrm{BI}$, high quality answers may be given. Because of that, BI can be used for testing of a hypothesis; it can even be used as a tool for clinical diagnosis. The breast imagingreporting and data system (BI-RADS) uses qualitative analysis and interoperations of medical doctors on the BI. By using 2D mammograms MDs breasts are classified based on thorax density as: (a) entirely fatty, (b) scattered areas of fibroglandular density, (c) heterogeneously dense, and (d) extremely dense. It is important to note that this method is based on MDs in 2D measures, and that it can be wrong if it is done in 3D systems [16-18]. Because of this, all the results have been done inside a $2 \mathrm{D}$ system, and measured in 2D working domain. BI-RADS breasts classified by density are shown in Fig. 4. As an elementary credibility of the image, it is important to note that the images were made in the same environment, and taken by the same camera, so that final results can be measured. Method of Hybrid edge detection has been confirmed as ideal in the field of detection/acknowledgment and affirmation of curves within nanoparticles, and as such has been experimentally applied to medical images in this paper.

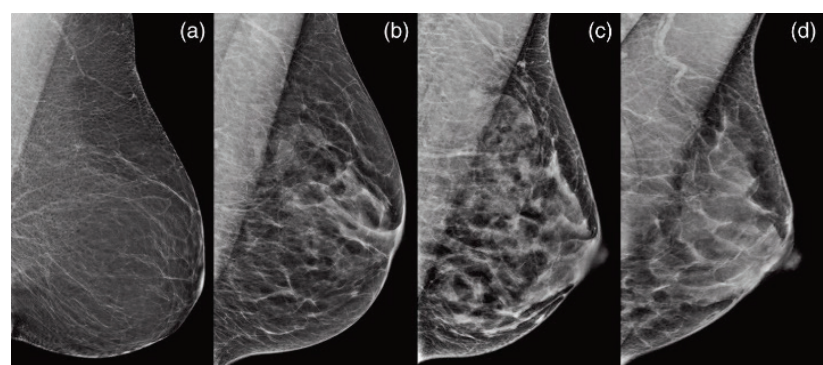

Figure 4 BI-RADS breasts classified by density (a) entirely fatty, (b) scattered areas of fibroglandular density, (c) heterogeneously dense, and (d) extremely dense

\section{SEGMENTATION WITH HYBRID EDGE DETECTION ALGORITHM}

Images used in this research are images which can be found in the medical image data base defined in papers [1924] as well as images which were available during the medical research at the Clinical Centre of Serbia. The degree of useful information makes the difference between the signals of original images and images treated after the segmentation. In this way, obtained results of processing (visual and numeric) are comparable with results obtained by other methods. In the case of this images data base, a new (suggested) method for image segmentation has been applied and it sublimes hybrid edge detection method (for original image and negative), weighted filter and specifically defined edge detection threshold.

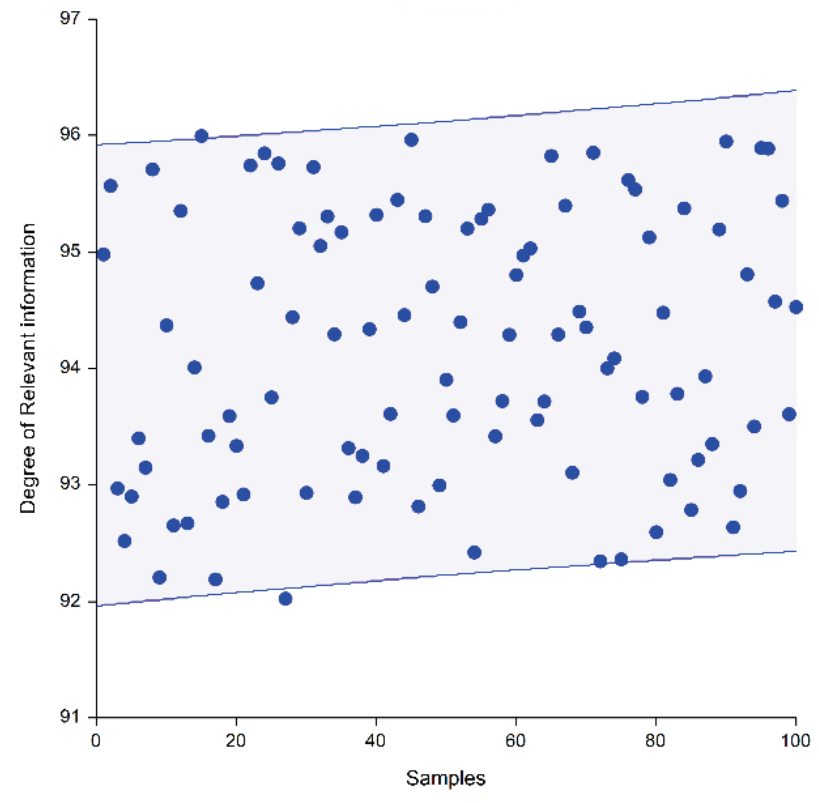

Figure $\mathbf{5}$ The degree of useful information in the observed samples afte applying Hybrid edge detection

After application of Hybrid edge detection algorithm on BIRADS breasts images, received edit has more than $93 \%$ of adequacy which carries useful information as has been defined in previous works [19-24]. Degree of useful information is based on concentration of changes on a certain part of an image. Results of the edit were shown oin Fig. 6. and the degree of useful information has been shown in Fig. 5. 


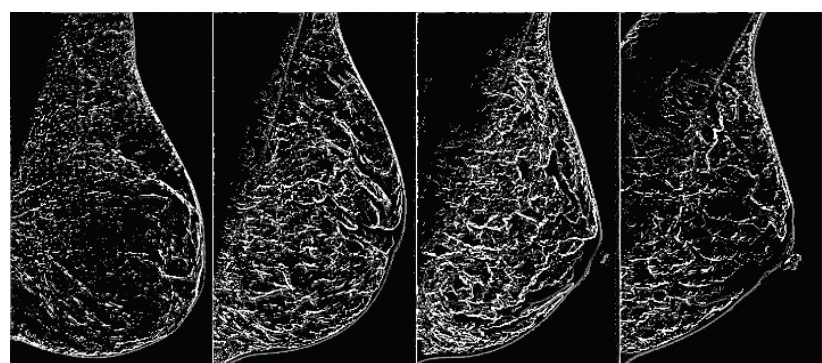

Figure 6 Original image processing by the Hybrid edge detection

Very important area of digital image processing is segmentation of a certain image. After the quality of the image, using the Hybrid edge detection, has been determined, the Segmentation Mosaic algorithm can be used. Results of the edit after segmentation have been shown in Fig. 6. The post-segmentation view gives the visually emphasized segments of the four breast density classes, which are now very suitable for further processing. In the next step, the level of details of the image can be measured for every density. The given values of the level of details will be connected to the already defined density classes. Quantitative results of the analysis will be treated based on a database of medical images. Given values for causes will be grouped inside a determined area with precision of $95 \%$.

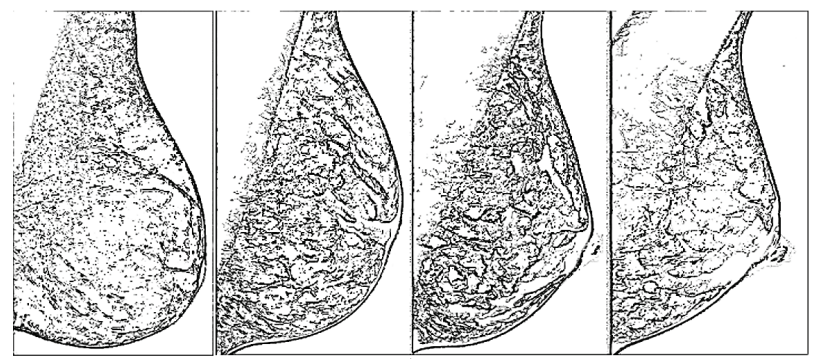

Figure 7 Original image processing by the Segmentation Mosaic method with Hybrid edge detection

\section{RESULTS \&DISCUSSION}

Connection of BI-RADS breasts classified by density, with concentration of the level of details in images is done on 100 medical images. Results have shown that the level of density BI-RADS breasts can be connected to the parameter of level of detail within digital images. Fig. 8 as another characteristic image shows the 4 defined types of breasts classified by density. Radiomics represents the process of generation databases of medical images that can be searched and that can be used for analysis of quantitative analysis [25]. This potential analysis of medical images gives a new dimension to image processing, as well as a base for further editing. With this base, it is possible to cross-search, combine or compare different elements to different images and in that way gain unique conclusions, especially when it concerns a database for one patient [26]. Repeated procedure of processing of Fig. 4, through Fig. 6 and 7, treated other images used as samples as well. In that way Fig. 9 came out of Fig. 8.

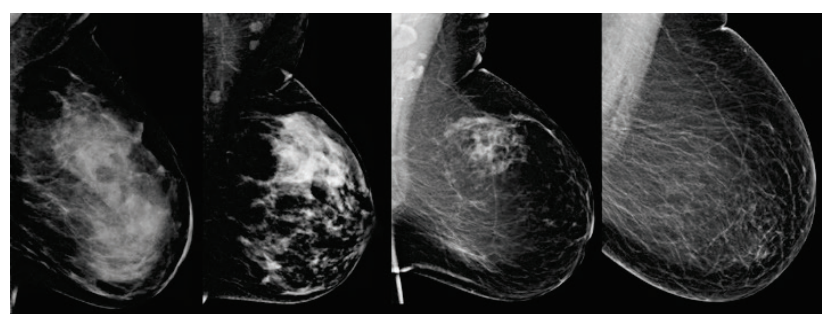

Figure 8 Original BI-RADS breasts classified by density (a) entirely fatty, (b) scattered areas of fibroglandular density, (c) heterogeneously dense, and (d) extremely dense

Values of level of detail of shown examples for entirely fatty were shown in Fig. 10. Those values for entirely fatty vary from 0,4 to 0,54 according to measures of discrete cosine transformations, which represents a low level of details. High level of fat can be treated as a low level of discontinuity on images, ergo the fact that there is a low level of details. Values of standard deviation for this type of density are circa $9 \%$. There were no extreme deviations on the segments so it can be said that the entirely fatty ones can almost always be treated under 0,55 values of the level of details for discrete cosine transformations. Scattered areas of fibroglandular density and heterogeneously dense ones as a class of middle density, have a respectively,middle level of details. For scatterd areas of fibroglandular density, level of details is from 0,62 to 0,77 , and for heterogeneously dense ones level of details is from 0,79 to 0,97 . Even if remotely divided, these fields are very close to one another. As there is no drastic deviation of value of any sample, two fields can be treated as referential.
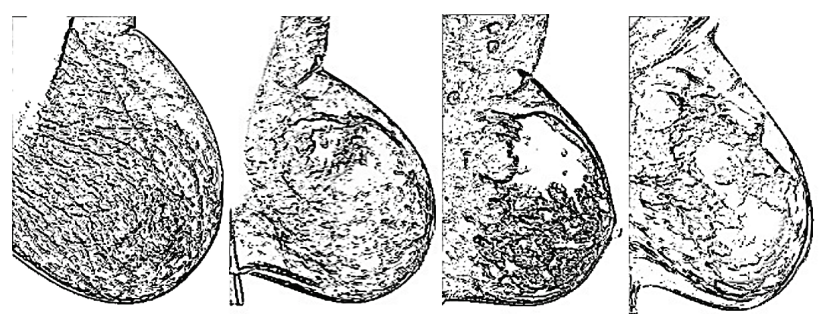

Figure 9 Images from Fig. 5 processing by the Segmentation Mosaic method with Hybrid edge detection

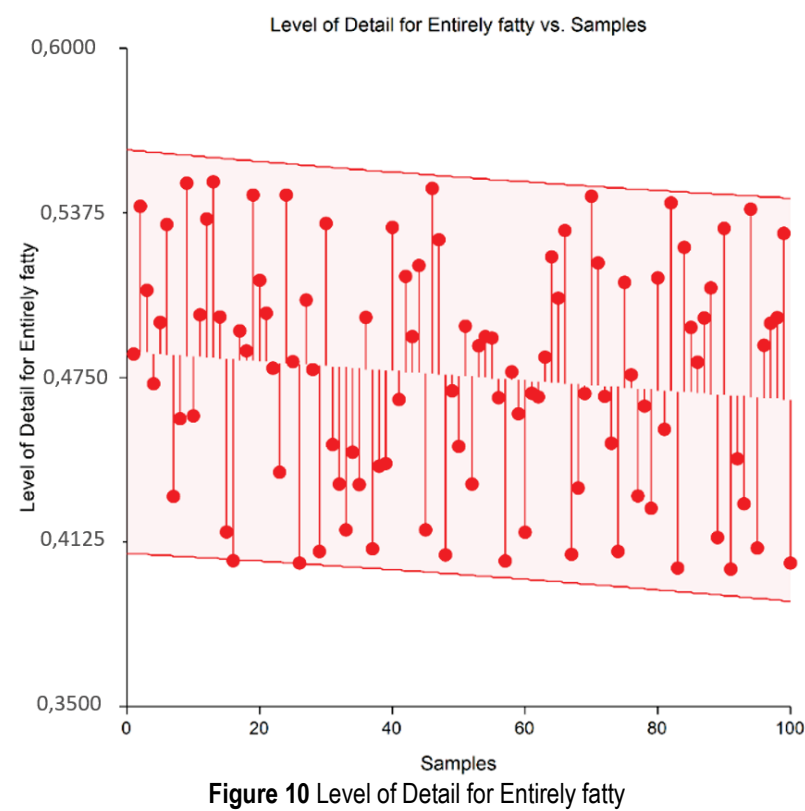




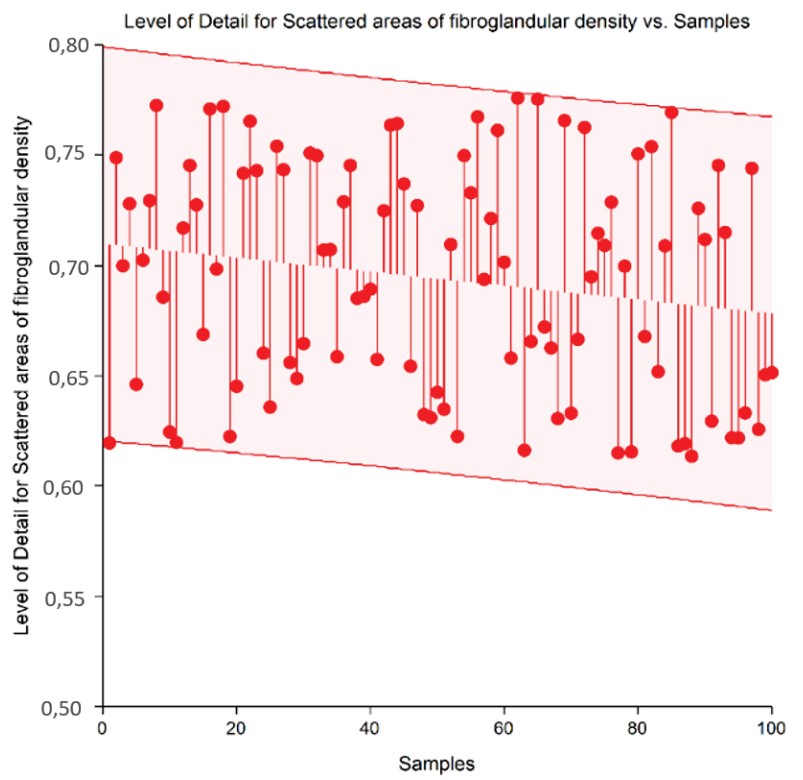

Figure 11 Level of Detail for Scattered areas of fibroglandular density

The highest level of details can be found within extremely dense ones-from 1,02 to 1,71 . There is the biggest deviation from the middle value, from the given extent of values, but without extreme deviations which can disturb the integrity of this field. An important characteristic in marking of the variations of a parameter in certain extent is the standard deviation. Values of different types of density are shown in Fig. 14. The highest level of standard deviation is level extremely dense, which is around $15 \%$ of average value of the observed field.

Analysed images samples show areas which contain parameters for LoD. Fig. 10, Fig. 11, Fig. 12 and Fig. 13 have no overlaps in levels of detail between defined areas. Due to this fact, there is no error in classification between the analysed areas.

Modelling of cancer using a computer is a field which is being developed; at this moment it is based on examination and analysis of a big number of medical images. In addition to this, results of using multiscale modelling of growing tumors had long term negative effects on phenotypes [27].

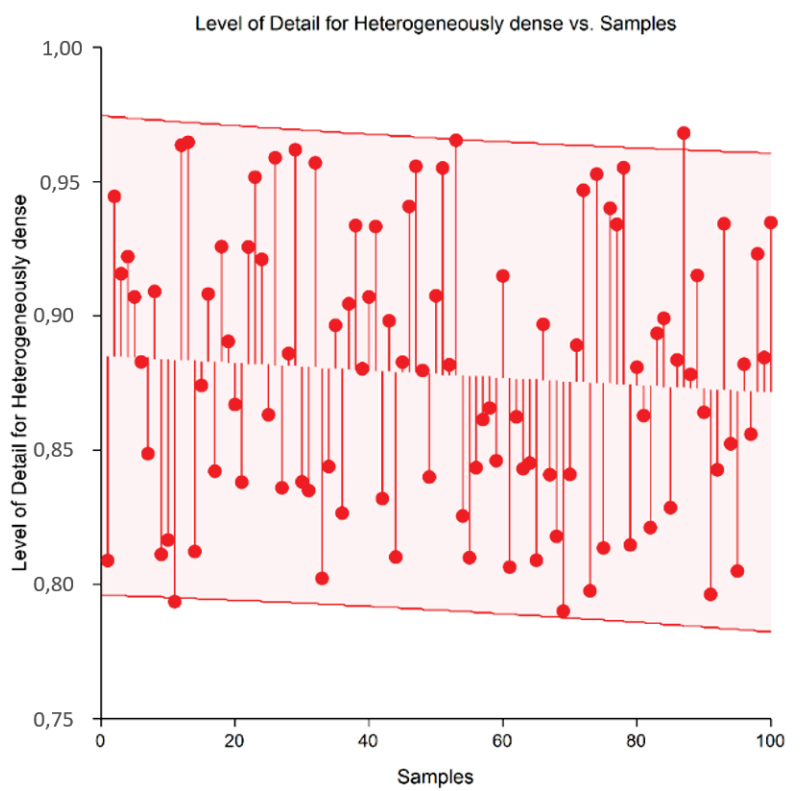

Figure 12 Level of Detail for Heterogeneously dense

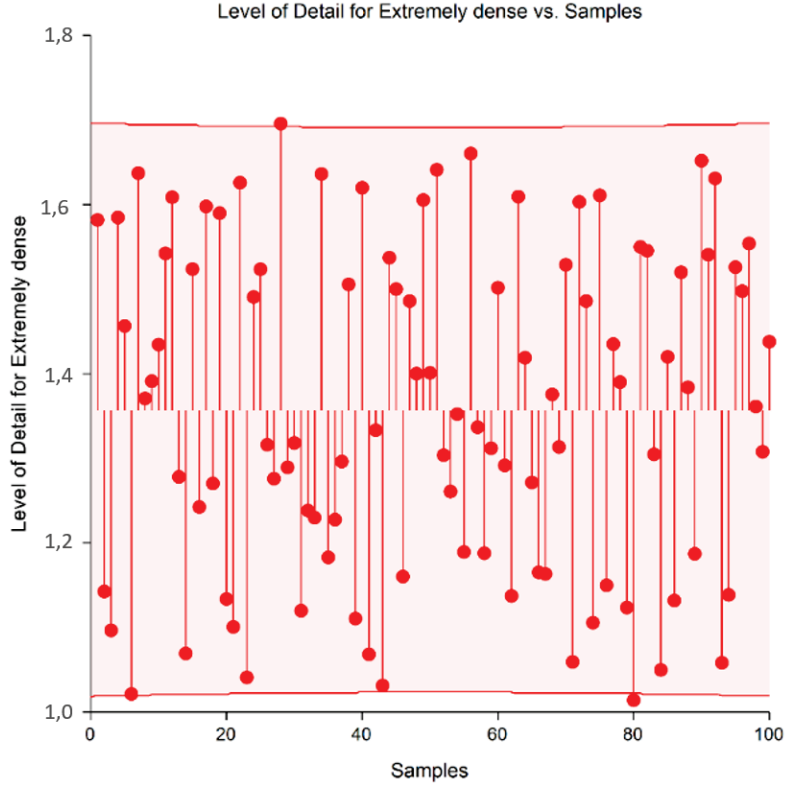

Figure 13 Level of Detail for Extremely dense

Conclusions about negative effects of experimentally verified models of breast cancer on phenotypes can be found in the paper [28]. Experiences and mistakes that have been approved and verified through the given works, limit the research in this paper in the conclusions. These reasons have, until this day, forbidden direct connection of segments of digital images with models of breast cancer, so this can be taken as the limit of this research.

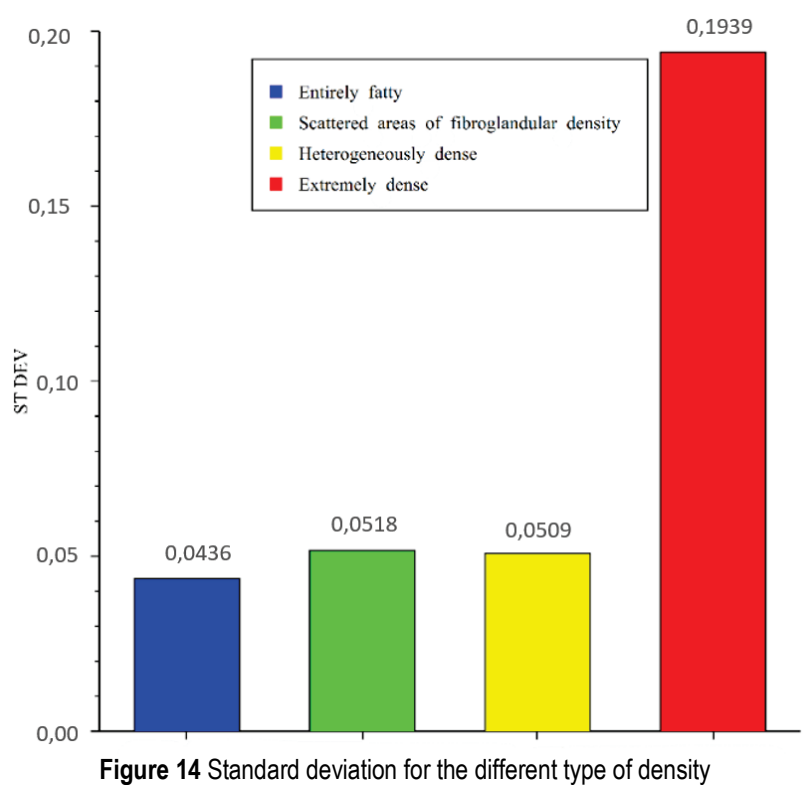

The described methods of segmentation in this paper are subjected to comparison with other methods of segmentation, described in papers [29-31]. Besides the stated advantages of the theoretical frame of digital image processing and approved using of mathematical tools, Fig. 15 provides visually comparative analysis of results of a few methods [31, 32], and Fig. 16 shows results received after edit of the attached method in this paper.

Methods shown in Fig. 15 are based on the texture analysis. Textures take into account a group of pixels and on the basis of that group, they form segments. The main disadvantage of these methods is that textures do not match 
all changes in images, and therefore they do not match analysed changes inside the breasts. Another disadvantage of these methods is that in transformations of $3 \mathrm{D}$ area into a 2D image there is often an error because a lot of layers of texture are shown in the same image. Existing methods form segments/regions on the basis of similarity of texture, and the suggested method treats each pixel separately.

a)
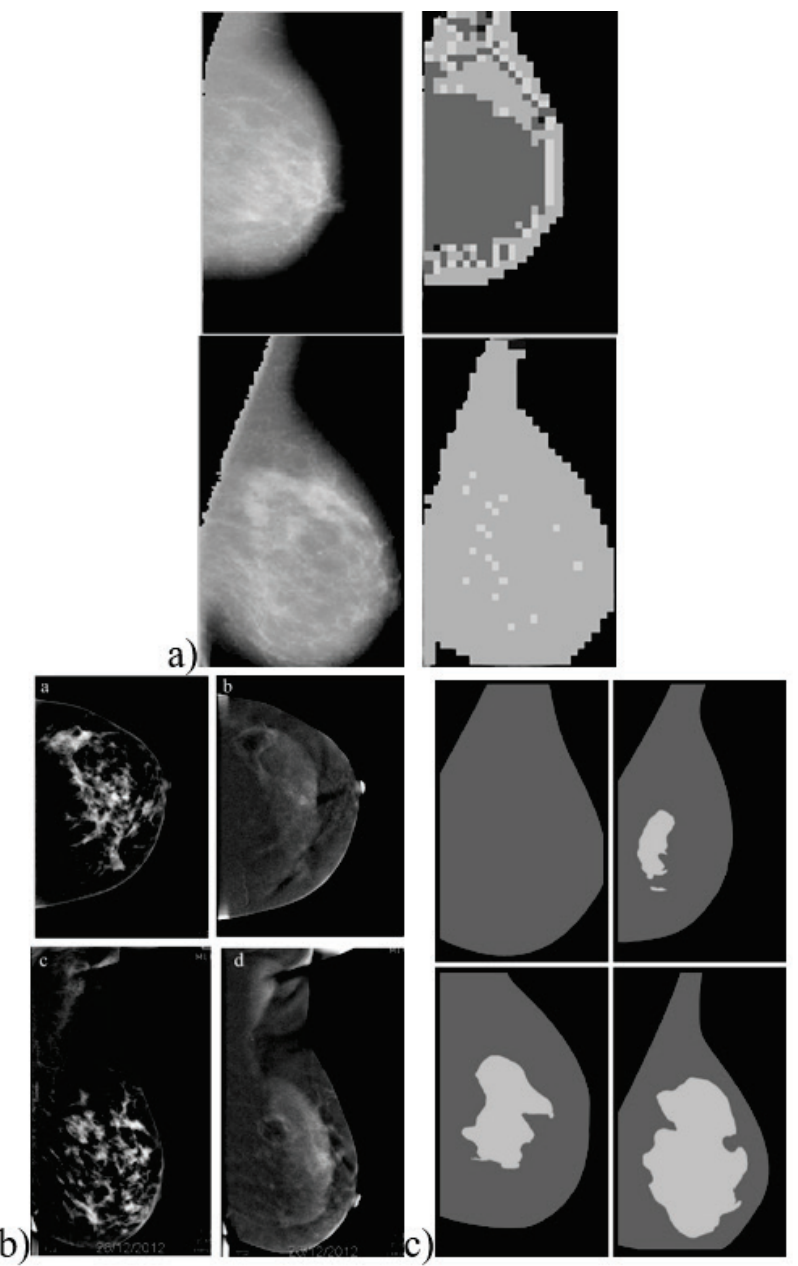

Figure $15 \mathrm{~A}$ visual representation of the comparative segmentation methods described in the manuscripts, respectively [29-31]

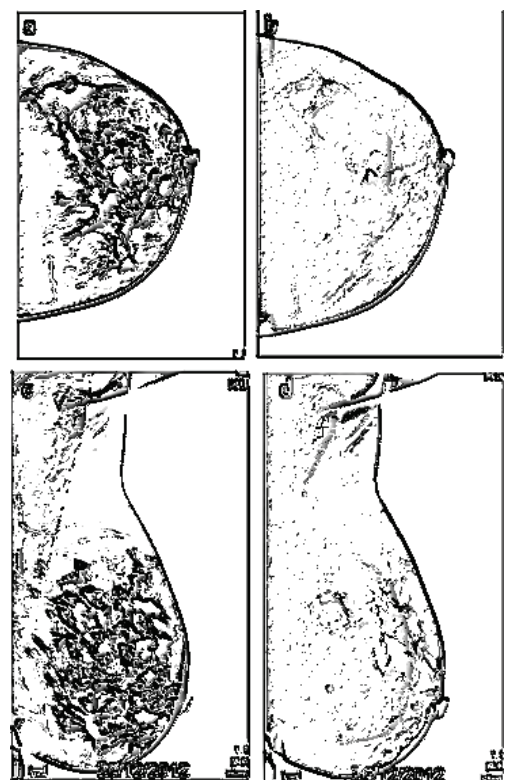

Figure 16 Visual representation of the segmentation by the proposed method in this paper
The method suggested in this paper detects every change because each pixel in the image is in the "centre of attention". In this way errors in segment detection are minimised as each pixel in the image is treated.

Methods of segmentation differ, which is clearly seen in the image. Proposed segmentation of medical images gives the widest spectre of detected elements in the image, which affirms the highest level of details detected within images that have been compared. Therefore, only the proposed method could relevantly connect with the fields of levels according to density. Other methods depend on parameters for detection of segmentation so as nonparametric methods they do not give results for connection to the level of details.

\section{CONCLUSION}

Applied way of image segmentation represents a new method and it is completely different from those used before. This paper clearly shows that the level of fats in breasts can be directly connected to the level of details within medical imaging. Highest level of fats will have extremely low level of details so it can be said that the level of details within digital imaging is inversely proportional to the density of all 4 types. Even if, at the time being, for the given reasons, connection of the mentioned segments of digital images with breast cancer models is not possible, this paper gives framework and algorithm conclusions for this issue. However, before clinical application of this or similar method, a lot of questions must be looked at, and conclusions must be made regarding digital imaging, databases, medical researches and therapies. Numerous advantages of the models give better performances to analysis of medicinal images of all types, within the same image generating speed. Contrary to other methods which treat groups of pixels as segments, the method suggested in this paper treats each pixel separately, which drastically decreases the possibility of error in segment detection. Research in this paper has connected the level of details of digital images after processing of medical images with Mosaic segmentation methods with Hybrid edge detection. Therefore, the results have experimentally shown that every density class can relatively connect with concrete level of details area. Also, complete independence of each part of algorithm means additional advantage for implementation of new ones if there is a need for it.

\section{Acknowledgment}

This work was done within the research project of the Ministry of Science and Technological Development of Serbia TR35026, III47016 and III44006.

\section{REFERENCES}

[1] Rajković, I. \& Žiljak, V. (2018). Parallel Motion Images in Visual and Near Infrared Spectrum. Tehnički vjesnik, 25(4), 1004-1008. https://doi.org/10.17559/TV-20160610125726

[2] Zhou, Y., Zhao, T., Wang, Y., Wu, J., \& Zhou, X. (2018). A Linear Fitting Density Peaks Clustering Algorithm for Image Segmentation. Tehnički vjesnik, 25(3), 808-812. https://doi.org/10.17559/TV-20171125161944

[3] Khalifa, T. \& Sengul, G. (2018). The Integrated Usage of LBP and HOG Transformations and Machine Learning 
Algorithms for Age Range Prediction from Facial Images. Tehnički vjesnik, 25(5), 1356-1362. https://doi.org/10.17559/TV-20170308030459

[4] Deserno, M. T., Handels, H., Maier-Hein, H. K., Mersmann, S., Palm, C., Tolxdorff, T., Wagenknecht, G., \& Wittenberg, T. (2013). Viewpoints on Medical Image Processing: From Science to Application. Current Medical Imaging Reviews, 9(2), 79-88. https://doi.org/10.2174/1573405611309020002

[5] Kass, M., Witkin, A., \& Terzopoulos, D. (1988). Snakes: Active contour models. International Journal Computer Vision, 1(4), 321-331. https://doi.org/10.1007/BF00133570

[6] Ozertem, U. \& Erdogmus, D. (2007). Nonparametric snakes. IEEE Transactions on Image Processing, 16(9), 2361-8. https://doi.org/10.1109/TIP.2007.902335

[7] Martin, P., Réfrégier, P., Galland, F., \& Guérault, F. (2006). Non parametric statistical snake based on the minimum stochastic complexity. IEEE Transactions on Image Processing, 15(9), 2762-70. https://doi.org/10.1109/TIP.2006.877317

[8] Kim, J., Çetin, M., \& Willsky, A.S. (2007). Nonparametric shape priors for active contour-based image segmentation. Signal Process, 87(12), 3021-3044. https://doi.org/10.1016/j.sigpro.2007.05.026

[9] Holst, G. C. \& Lomheim, T. S. (2007). CMOS/CCD Sensors and Camera Systems. Bellingham. WA, USA, SPIE PRESS BOOK.

[10] Xuelong, L., Dacheng, T., Xinbo, G. \& Wen, L. (2009). A natural image quality evaluation metric. Signal Processing, 89(4), 548-555. https://doi.org/10.1016/j.sigpro.2008.10.007

[11] Wang, Z., Bovik, A. C., \& Lu, L. (2002). Why is Image Quality Assessment So Difficult? Proceedings of ICASSP. 1, 1520-6149. https://doi.org/10.1109//CASSP.2002.5745362

[12] Shnayderman, A., Gusev, A., \& Eskicioglu, A. M. (2006). An SVD-Based Gray-Scale Image Quality Measure for Local and Global Assessment. IEEE Transactions on Image Processing, 15(2), 422-429. https://doi.org/10.1109/TIP.2005.860605

[13] ITU-T. (2004). TUTORIAL: Objective perceptual assessment of video quality, Full reference television.

[14] Ivkovic, R., Petrović, M., Spalević, P., \& Miljković, D. (2014). Analysis of quality of nonlinear filters by removing salt \& pepper noises. YU INFO 2014, Serbia, Kopaonik, Feb. 2014, 330-333

[15] Ivković, R., Milošević, I., Gara, B., Minić, S., \& Miljković, D. (2014). Rekonstrukcija slika vidljivog spektra snimljenih sa malim procentom osvetljenja. XIII medunarodni naucnostrucni simpozijum INFOTEH 2014, 13(1), 648-651. https://infoteh.rs.ba/rad/2014/RSS-2/RSS-2-1.pdf

[16] Sartor, H., Lång,K., Rosso, A., Borgquist, S., Zackrisson, S., \& Timberg P. (2016). Measuring mammographic density: comparing a fully automated volumetric assessment versus European radiologists qualitative classification. European Radiology, 26(12), 4354-4360. https://doi.org/10.1007/s00330-016-4309-3

[17] Eom, H. J., Cha, J. H., Kang, J. W., Choi, W. J., Kim, H. J., $\&$ Go, E. (2018). Comparison of variability in breast density assessment by BI-RADS category according to the level of experience. Acta Radiologica, 59(5), 527-532. https://doi.org/10.1177/0284185117725369

[18] Gard, C. C., Aiello Bowles, E. J., Miglioretti, D. L., Taplin, S. H., \& Rutter, C. M. (2015). Misclassification of Breast Imaging Reporting and Data System (BI-RADS) Mammographic Density and Implications for Breast Density Reporting Legislation. The breast journal, 21(5), 481-9. https://doi.org/10.1111/tbj.12443

[19] Larin, K. V. \& Sampson, D. D. (2017). Optical coherence elastography OCT at work in tissue biomechanics. Biomedical Optics Express, 8(2), 1172-1202. https://doi.org/10.1364/BOE.8.001172
[20] Nolan, M. R., Adie, G. S., Marjanovic, M., Chaney, J. E., South, A. F., Monroy, L. G., Shemonski, D. N., EricksonBhatt, J. S., Shelton, L. R., Bower, J. A., Simpson, G. D., Cradock, A. K., Liu, G. Z., Ray, S. P., \& Boppart, A. B. (2016). Intraoperative optical coherence tomography for assessing human lymph nodes for metastatic cancer. $B M C$ Cancer, 16(1), 1-10. https://doi.org/10.1186/s12885-016-2194-4

[21] Liang, C. P., Yang, B., Kim, I. K., Makris, G., Desai, J. P., Gullapalli, R. P., \& Chen, Y. (2013). Concurrent multiscale imaging with magnetic resonance imaging and optical coherence tomography. Journal of Biomedical Optics, 18(4), 040506. https://doi.org/10.1117/1.JBO.18.4.046015

[22] Yao, J., Xia, J., \& Wang, L. V. (2015). Multiscale Functional and Molecular Photoacoustic Tomography. Ultrasonic imaging, 38(1), 44-62. https://doi.org/10.1177/0161734615584312

[23] Kang, J., Kim, E. K., Kim, G. R., Yoon, C., Song, T. K., \& Chang, J. H. (2015). Photoacoustic imaging of breast microcalcifications: a validation study with 3-dimensional ex vivo data and spectrophotometric measurement. Journal of Biophotonics, 8(1-2), 71-80. https://doi.org/10.1002/jbio.201300100

[24] Akers, W. J., Edwards, W. B., Kim, C., Xu, B., Erpelding, T. N., Wang, L. V., \& Achilefu, S. (2011). Multimodal sentinel lymph node mapping with single-photon emission computed tomography (SPECT)/computed tomography (CT) and photoacoustic tomography. Translational research: the journal of laboratory and clinical medicine, 159(3), 175-81. https://doi.org/10.1016/j.trsl.2011.09.006

[25] Gillies, J. R., Kinahan, E. P., \& Hricak, H. (2015). Radiomics: images are more than pictures, they are data. Radiology, 278(2), 563-577. https://doi.org/10.1148/radiol.2015151169

[26] Dinov I. D. (2016). Methodological challenges and analytic opportunities for modeling and interpreting Big Healthcare Data. GigaScience, 5(12), 1-15. https://doi.org/10.1186/s13742-016-0117-6

[27] Robertson-Tessi, M., Gillies, J. R., Gatenby, A. R., \& Anderson, R. A. A. (2015). Impact of Metabolic Heterogeneity on Tumor Growth, Invasion, and Treatment Outcomes. Cancer Research, 75(8), 1567-1579. https://doi.org/10.1158/0008-5472.CAN-14-1428

[28] Simmons, A., Burrage, M. P., Nicolau Jr, V. D., Lakhani, R. S., \& Burrage K. (2017). Environmental factors in breast cancer invasion: a mathematical modelling review. Pathology, 49(2), 172-180. https://doi.org/10.1016/j.pathol.2016.11.004

[29] Pahwa, S., Hari, S., Thulkar, S., \& Angraal, S. (2015). Evaluation of breast parenchymal density with QUANTRA software. The Indian journal of radiology \& imaging, 25(4), 391-6. https://doi.org/10.4103/0971-3026.169458

[30] Bosch, A., Marti, J., Oliver, A., \& Munoz, X. (2006). Modeling and Classifying Breast Tissue Density in Mammograms. IEEE Computer Society Conference on Computer Vision and Pattern Recognition (CVPR'06), New York, 1552-1558. https://doi.org/10.1109/CVPR.2006.188

[31] Oliver, A., Lladó, X., Pérez, E., Pont, J., Denton, E. R., Freixenet, J., \& Martí, J. (2009). A statistical approach for breast density segmentation. Journal of digital imaging, 23(5), 527-37. https://doi.org/10.1007/s10278-009-9217-5

[32] Mokhtar, O. \& Mahmoud, S. (2014). Can contrast enhanced mammography solve the problem of dense breast lesions? Egyptian Journal of Radiology and Nuclear Medicine, 45(3), 1043-1052. https://doi.org/10.1016/j.ejrnm.2014.04.007 


\section{Contact information:}

Ratko IVKOVIĆ, PhD candidate,

(Corresponding author)

University of Priština,

Faculty of Technical Sciences,

Kneza Miloša 7, Kosovska Mitrovica, Serbia

phone: +38166946 4078

E-mail: ratko.ivkovic@pr.ac.rs

Mile PETROVIĆ, Full professor,

University of Priština,

Faculty of Technical Sciences,

Kneza Miloša 7, Kosovska Mitrovica, Serbia

E-mail: mile.petrovic@pr.ac.rs

Branislav DAKOVIĆ, Associate professor,

KBC „Dr Dragiša Mišović-Dedinje“,

Heroja Milana Tepića 1, Beograd, Srbija

E-mail: branislav.dakovic@dragisamisovic.bg.ac.rs

Branimir JAKŠIĆ, Assistant professor,

University of Priština,

Faculty of Technical Sciences,

Kneza Miloša 7, Kosovska Mitrovica, Serbia

E-mail: branimir.jaksic@pr.ac.rs

Ivana MILOŠEVIĆ, Lecturer

The School of Electrical and Computer Engineering,

Audio and video technologies

Vojvode Stepe 283, Belgrade, Serbia

E-mail: ivana.milosevic@viser.edu.rs 